\title{
Violencia Feminicida en Médicas Pasantes de Servicio Social12
}

\author{
Marisol Silva Vera \\ PhD. en Ciencias Médicas \\ Universidad de Guanajuato, México \\ Correo electrónico: msol78@gmail.com

\section{María de Jesús Jiménez González \\ $\mathrm{PhD}$. en Ciencias de Enfermería Universidad de Guanajuato, México Correo electrónico: mi.jimenez@ugto.mx}

Recibido: 26/08/2019

Evaluado: 31/01/2020

Aceptado: 13/02/2020

\section{Resumen}

Más de la mitad de los médicos pasantes no se perciben seguros en los Centros de Salud (CS), ya que se tienen registros de violencia en los CS, principalmente hacia las Mujeres Médicas Pasantes de Servicio Social (MPSS). El objetivo del estudio fue identificar, por medio de una encuesta, el tipo de violencia ejercido en una muestra de 60 mujeres de entre 22 a 24 años. Los resultados encontraron 4 situaciones de riesgo: inseguridad (30\%), vida en peligro (50\%), intento de irrupción (20\%) y tentativa de acoso sexual (30\%); además, se hallaron 6 diferentes tipos de violencia: psicológica (42.17 \pm 11.54), física (94.86 \pm 7.07$)$, económica $(83.45 \pm 5.24)$, sexual $(27.38 \pm 3.53)$, laboral $(53.13 \pm 36.05)$ y docente $(43.77 \pm 23.49)$. En las MPSS son evidentes las agresiones violentas, sugiriendo que los CS no tienen medidas de seguridad apropiadas para resguardar la seguridad de las MPSS.

Palabras clave Médicos pasantes, centros de salud, servicio social, inseguridad, violencia feminicida.

12 Para citar este artículo: Silva-Vera, M. y Jiménez-González, M.J. (2021). Violencia Feminicida en Médicas Pasantes de Servicio Social. Informes Psicológicos, 21(2), pp. 193-207 http://dx.doi.org/10.18566/infpsic.v21n2a12 


\title{
Femicidal Violence in Social Service Medical Interns
}

\begin{abstract}
More than half of medical interns do not feel safe in Health Centers (HS), since there are records of violence mainly towards social service female medical interns (SSFMI) in HS. The objective of the study was to identify the type of violence exercised in a sample of 60 women between 22 and 24 years old. The survey helped to identify four risk situations: insecurity $(30 \%)$, life in danger $(50 \%)$, attempted break-in (20\%) and attempted sexual harassment (30\%). Results also show six different types of violence: psychological (42.17 \pm 11.54 ), physical (94.86 \pm 7.07$)$, economic (83.45 \pm 5.24 ), sexual $(27.38 \pm 3.53)$, work $(53.13 \pm 36.05)$ and educational $(43.77 \pm 23.49)$. Violent assaults are evident in the SSFMI, suggesting that the HS do not have appropriate security measures to protect the safety of the SSFMI.
\end{abstract}

Keywords

Medical interns, health centers, social service, insecurity, femicidal violence.

\section{Violência feminicida em estagiários médicos do serviço social}

\section{Resumo}

Mais da metade dos estagiários médicos não se sentem em segurança nos Centros de Saúde (CS), visto que há registros de violência no CS, principalmente contra mulheres internas do Serviço Social (MPSS). 0 objetivo do estudo foi identificar, por meio de inquérito, o tipo de violência exercida em uma amostra de 60 mulheres entre 22 e 24 anos. Os resultados identificaram 4 situações de risco: insegurança (30\%), vida em perigo (50\%), tentativa de arrombamento $(20 \%)$ e tentativa de assédio sexual (30\%); Além disso, foram identificados 6 tipos diferentes de violência: psicológica (42.17 \pm 11.54$)$, física ( $94.86 \pm 7.07)$, econômica ( $83.45 \pm 5.24)$, sexual ( $27.38 \pm 3.53)$, laboral (53.13 $\pm 36.05)$ e educacional (43.77 \pm 23.49 ). Ataques violentos são evidentes no MPSS, sugerindo que os CS não possuem medidas de segurança adequadas para proteger a segurança do MPSS.

\section{Palavras chave} Médicos internos, centros de saúde, serviço social, insegurança, violência feminicida. 


\section{ntroducción}

La primera investigación científica, cualitativa y cuantitativa, sobre violencia de género contra las mujeres realizada en México, desde una perspectiva feminista de género y derechos humanos de las mujeres, investigó la condición social y la situación vital de las mujeres en cada entidad federativa a través de la metodología del enfoque del Desarrollo Humano con sus Índices de Género, encontrando que la violencia feminicida es una dimensión extraordinariamente poderosa de las formas de dominación de género de los hombres sobre las mujeres (Lagarde, 2001).

Asimismo, Ramírez et al. (2012) reportaron un aumento de la inseguridad en los centros de salud (CS) refiriendo que más de la mitad de los MPSS no se perciben seguras en los CS del país. Además, 1 de cada 4 relató que su vida corrió peligro en algún momento, el 35.9\% mencionó intentos de intrusión sin consentimiento al CS y, finalmente, 1 de cada 10 pasantes señaló de manera contundente tentativa de acoso o abuso sexual.

Diversos investigadores refieren una prevalencia de abuso de estudiantes en algún momento durante su formación, reportándose abuso en estudiantes de Medicina de los Estados Unidos de hasta un 98.9\%. En diversos países del mundo como Australia, Nueva Zelanda, Finlandia, Irlanda, Argentina, México, Colombia, Chile, Pakistán, Canadá y Japón, las tasas de abuso percibido entre estudiantes de Medicina oscilan entre el
25\% y el 89\% (Alzahrani, 2012; Benya et al., 2018; Hasty et al., 2018; Ritu \& Febbraro, 2013).

Los investigadores japoneses afirman que, en la universidad, el acoso a menudo puede ocurrir y permanecer sin ser expuesto, lo que desalienta a las jóvenes a seguir su carrera; además, concluyen que es necesario estimar y mejorar la percepción de "acoso escolar" entre las facultades universitarias (Nagasawa et al., 2019). En 1982, Silver publicó el primer estudio sobre violencia en estudiantes de Medicina y describió el estado de ánimo y deterioro de salud en los estudiantes debido al abuso que sufrieron, afirmando que posteriormente se volvieron "cínicos, desanimados, asustados, deprimidos o frustrados" (p. 29).

Hasta la fecha, se han llevado a cabo numerosas investigaciones en todo el mundo, informando la existencia de conducta feminicida contra MPSS, que se entiende como cualquier acto que perjudica la dignidad o la autoestima de las mujeres MPSS a nivel físico, psicológico y/o sexual. Este proceso implica ejercer relaciones de poder asimétricas que se traducen en diferentes formas de dominación/subordinación entre grupos (Izquierdo, 1998). Asimismo, FernándezMoreno en 2007 observó que existe preocupación e introspección en estudiantes con respecto a su propia situación como mujeres frente a la violencia, y esto se relacionó con los aspectos culturales, familiares y sociales de los contextos donde son originarios, pero también con su procedencia social y condición étnica.

Tanto las médicos mujeres como los hombres reportan altas tasas de violencia verbal, física y emocional, 
encontrando que es ligeramente más alta la violencia física y verbal entre los hombres, pero las mujeres sufren más violencia feminicida (Bonilla-Neira, Gómez-Meneses, \& Godoy-Pinilla, 2019; Moreno-Tetlacuilo et al., 2016), mientras que las mujeres muestran la prevalencia más alta, independientemente de su origen étnico (Nora et al., 2007). Rees y Monrouxe (2011) identificaron incidencia de violencia en médicos donde el 9.4\% de los hombres reporta acoso racial, mientras que el $16.8 \%$ de las mujeres refirieron violencia de género.

Discriminación de género y sexismo es el tipo más común de violencia feminicida. Así, Fnais et al. (2014) identificaron en un metanálisis una prevalencia significativamente mayor en mujeres que en hombres ( $p<.05)$; estas modalidades de violencia se expresan como comentarios despectivos (11.5\%) y/o insultos sexistas (3.4\%). Dentro de las disposiciones generales del Diario Oficial de la Federación (DOF), el servicio social de la carrera de Medicina se realizará de acuerdo con la Ley Reglamentaria del Artículo 5o. Constitucional y la Ley General de Salud. El DOF señala además que los aspectos académicos quedan a cargo de las Universidades y de las Instituciones de Salud y éstas determinarán los programas operativos, así como las áreas del campo clínico. Respecto al periodo para realizar el servicio social, la Ley General de Salud refiere "el servicio social se ejecutará durante doce meses continuos" y las áreas urbanas y rurales con menor desarrollo económico y social, serán los lugares donde se ejecute dicho servicio social. Relativo a la seguridad, el DOF menciona que las instituciones de salud a la que pertenezca el campo clínico y la institución educativa que lo ocupe, deberán establecer políticas públicas con los actores sociales para salvaguardar la integridad física de los y las pasantes.

Los documentos relacionados al servicio social en los apartados sobre derechos y obligaciones del pasante de Medicina destacan que deberá prestar atención médica, realizar el total de las acciones de salud, cumplir al 100\% el contenido de los programas académicos, programas operativos y realizar un diagnóstico de salud comunitario donde se integre el apartado de infraestructura, equipamiento y abasto de insumos al inicio durante la prestación del servicio social (Secretaría de Salud, 2019). EI MPSS deberá informar a las autoridades de la comunidad, a la Institución de Salud y a su Universidad, cualquier incidente que ocurra relacionado a sus funciones y cuando se presente algún incidente durante la prestación del servicio social. Los documentos estipulan que los MPSS serán tratados de forma atenta y respetuosa por sus superiores, iguales o subalternos, siempre deberán ser escuchados por sus autoridades respectivas. Es importante especificar que en los documentos no se hace referencia sobre la diferencia de derechos en hombres ni mujeres.

Se sabe que los médicos-docentes a cargo de los internos de pregrado ejercen total autoridad sobre ellos, esto señalado claramente en los programas académicos y operativos oficiales de cada una de las Universidades de las cuales provienen los estudiantes. Los reportes en contra del autoritarismo médico sobre los estudiantes son indiscutibles, no obstante, esta condición es tolerada por el $100 \%$ de la comunidad médica. Los jefes de enseñanza tienen información 
de los testimonios de médicas internas donde los superiores han efectuado algún tipo de violencia hacia ellas, siendo la violencia psicológica la más reportada, conductas que en algunas ocasiones se torna en violencia feminicida (Frank, Carrera, Stratton, Bickel, \& Nora, 2006; López, González \& Velasco, 2004). Vanlneveld et al. (1996) informaron que el 50\% de los estudiantes acosados eran mujeres agredidas por otros médicos, y que, durante los primeros años de la escuela de Medicina, los perpetradores son pares. Asimismo, Mavis, Sousa, Lipscomb y Rappley (2014) observaron diferencias estadísticamente significativas en el cuarto y quinto año de la carrera de medicina, refiriendo que las situaciones de acoso y abuso sexual reportadas aumentan con el número de años de estudio.

Otros estudios en población médica mexicana afirman que algunos hombres con posición de poder son los principales perpetradores, especialmente de la administración de las clínicas, residentes o médicos especialistas del Departamento de Cirugía, seguido por profesores o instructores de ciclo básico, así como personal de enfermería y, en menor medida, otros pasantes o estudiantes (Castro, 2014).

La Asociación Mexicana de Facultades y Escuelas de Medicina (AMFEM) también señala que es responsabilidad y obligación de las IES retirar al MPSS de las sedes asignadas cuando las condiciones de vivienda, seguridad o atención no sean las indicadas en relación con la Norma correspondiente. La función de la supervisión médica en los CS consiste en vigilar y guiar a los subordinados de tal forma que las actividades se realicen adecuadamente, y será la inspección de un trabajo o actividad por un superior que permite la medición de la actuación y el control de las acciones. Las condiciones de supervisión hacia los pasantes de servicio social son precarias, sin planeación, control ni mucho menos retroalimentación, en un contexto de total de autoritarismo desmedido por parte nuevamente de las autoridades médicas y que los reportes la mayoría de las ocasiones quedan en un formato más, llenado y visto.

Asimismo, Ramírez (2009) refiere que el ambiente en el que los pasantes desarrollan su servicio social no corresponde a las expectativas que tienen sobre su futuro profesional y la adaptación al nuevo contexto les resulta difícil debido a que se encuentran lejos de su ambiente cultural y social, la mayoría de los internos de pregrado desean desempeñar su servicio social en el tercer o cuarto nivel de atención y solo un reducido $11.1 \%$ muestra alguna inclinación por el primer nivel. Los casos recientes de acoso sexual ilustran que tales experiencias también ocurren en las escuelas de Medicina. Se desconoce cuántas MPSS han experimentado directamente algún tipo de violencia. Las denuncias de las MPSS en diversos poblados de la república despertaron la inquietud de indagar más sobre este tema, con el objetivo de identificar la prevalencia de los tipos de violencia ejercido sobre las MPSS dentro de un periodo del 2012 al 2013, así como fomentar la creación de políticas públicas en favor de las MPSS.

La violencia feminicida es entonces una forma extrema de violencia de género contra las mujeres, producto de la violación de sus derechos humanos de manera reiterada y sistemática en los ámbitos público y privado, conformada por el conjunto de conductas misóginas que pueden conllevar impunidad social y del 
Estado y puede culminar en homicidio y otras formas de muerte violenta de mujeres (Lagarte, 1996).

Así, Bullen y Diez (2008), en su libro "Retos teóricos y nuevas prácticas", afirman que el feminicidio se consuma porque las autoridades omisas, negligentes o coludidas con agresores, ejercen sobre las mujeres violencia institucional al obstaculizar su acceso a la justicia y con ello contribuyen a la impunidad.

Por todo esto, el objetivo del estudio fue identificar el tipo de violencia ejercido en una muestra de 60 mujeres médicas pasantes de servicio social.

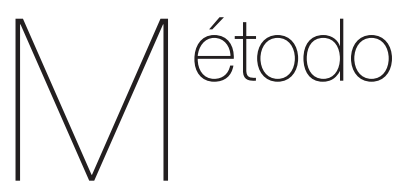

Se diseñó un estudio de tipo descriptivo y transversal dado que, en apego al objetivo de investigación, se pretendió identificar el tipo de violencia feminicida ejercicio sobre las MPSS caracterizando y categorizando eventos (Ato, López \& Benavente, 2013).

\section{Participantes}

Por medio de muestreo no probabilístico, de tipo intencional, se incluyó una muestra de 60 MPSS inscritas al servicio social en el periodo comprendido del 2012-2013, las cuales pertenecen a diferentes universidades, con una edad de entre 22 a 24 años y asignadas en diferentes CS del Estado de Guanajuato, tanto rurales como urbanos. Se solicitó a todas las participantes que contestaran un cuestionario después de consentir en participar en el estudio por escrito; además, se les aseguró la confidencialidad de los datos y fueron libres de retirarlos en cualquier momento durante el estudio, en consonancia con la Declaración de Helsinki sobre investigación en humanos y la Ley General de Salud. El estudio fue aprobado por el comité de ética institucional con el no. R192017.

Se aplicó una encuesta titulada "Acoso Académico" diseñada por las autoras y basada en la revisión de publicaciones realizadas en el tema, para establecer una definición conceptual de la violencia feminicida y las situaciones de riesgo en MPSS. El instrumento fue sometido a una prueba piloto en 80 estudiantes de Medicina de diversos años, seleccionados de manera aleatoria, obteniendo un alfa de Cronbach de .87. La encuesta que se diseñó incluía 4 secciones: 1) Características sociodemográficas y académicas; 2) Violencia feminicida, que contenía 4 ítems para cada dimensión: a) psicológica, b) física, c) docente, d) sexual, e) laboral y f) económica; 3) Situaciones de Riesgo, con 2 ítems para cada dimensión: a) inseguridad, b) vida en peligro, c) intento de irrupción, y d) tentativa de acoso; 4) Experiencias de riesgo: se describió la experiencia y si denunciaron el tipo de violencia sufrida. Las respuestas al cuestionario se calificaron en una escala del 1 al 5 , donde $1=$ nunca y $5=$ con frecuencia. Se utilizaron distribuciones de frecuencias, medias y desviación estándar para las variables socio-demográficas, así como un análisis de regresión lineal para estimar las variables relacionadas al género, violencia feminicida y situaciones de riesgo en el CS. Se aplicó el paquete estadístico SPSS en 
la versión 21 (Windows 98, SPSS México S.A de C.V).

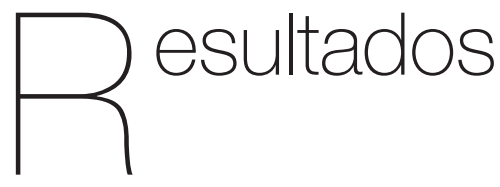

Características de las Médicas Pasantes de Servicio Social

Las 60 MPSS fueron entrevistadas y encuestadas en un ambiente propicio de tribuna libre, identificando una edad de entre $22.86 \pm 2.82$ años, escolaridad $21.08 \pm 1.5$ años; estado civil: casada $1.6 \%$, soltera $93.3 \%$, y unión libre .5\%. El 58.3\% de las MPSS se encontraba asignada en un CS rural, mientras que el 41.25\% en un CS urbano (ver Tabla 1).

Tabla 1

Características Sociodemográficas de las Médicas Pasantes

\begin{tabular}{lc}
\hline Variable & Mujeres MPSS* $(\mathbf{n}=\mathbf{6 0})$ \\
\hline Edad (años) & $22.86 \pm 2.82$ \\
Escolaridad (años) & \\
Estado Civil (\%) & 1.6 \\
$\quad$ Casada & 93.3 \\
Soltera & .5 \\
$\quad$ Unión Libre & \\
Tipo de Centro de Salud (\%) & \\
Rural & 58.30 \\
Urbano & 41.25 \\
Tipo de Universidad (\%) & \\
Privada & 48 \\
Pública & 52 \\
\hline
\end{tabular}

Fuente: Entrevista dirigida a MPSS. Los datos son expresados como media \pm desviación estándar y porcentaje \%. ${ }^{*}$ MPSS: Médicos Pasantes de Servicio Social.

\section{Situaciones de Riesgo identificadas en los Centros de Salud del Estado de Guanajuato}

La carencia de seguridad fue evidente, ya que las MPSS no estuvieron libres de inseguridad. Las situaciones de riesgo identificadas fueron: inseguridad en el centro de salud (30\%), vida en peligro en algún momento (50\%), intento de irrupción al centro de salud (20\%) y tentativa de acoso sexual (30\%). Algunas pasantes de servicio social refirieron más de una situación de riesgo en su CS (ver Tabla 2). Esto indica que las autoridades no se mostraron interesadas en salvaguardar la seguridad de las MPSS incluso sabiendo que eran mujeres solas que se enfrentaban a lugares probablemente peligrosos.

Tabla 2.

Situaciones de Riesgo Identificados en los Centros de Salud del Estado de Guanajuato

\begin{tabular}{lc}
\hline Tipo de Violencia Feminicida $^{\mathrm{a}}$ & Mujeres MPSS* $^{\star}(\mathbf{=} \mathbf{6 0})$ \\
\hline Psicológica & $42.17 \pm 11.54$ \\
Física & $94.86 \pm 7.07$ \\
Económica & $83.45 \pm 5.24$ \\
Sexual & $27.38 \pm 3.53$ \\
Laboral & $53.13 \pm 3.05$ \\
Docente & $43.77 \pm 2.49$ \\
\hline
\end{tabular}

Fuente: Entrevista dirigida a MPSS. Los datos son expresados en porcentaje $\%$. ${ }^{a}$ El total es mayor al $100 \%$ porque algunas pasantes reportaron más de una forma de abuso. *MPSS: Médicos Pasantes de Servicio Social.

\section{Tipo de Violencia Feminicida identificada en los Centros de Salud del Estado de Guanajuato}

Las acciones y/o omisiones que causaron daño y/o sufrimiento en las MPSS en las diferentes CS rurales y urbanos del Estado de Guanajuato, se identificaron 
como violencia psicológica (42.17 \pm 11.54), física (94.86 \pm 7.07$)$, económica (83.45 \pm 5.24$)$, sexual (27.38 \pm 3.53$)$, laboral $(53.13 \pm 36.05)$ y docente $(43.77 \pm$ 23.49). Algunas pasantes de servicio social refirieron más de un tipo de violencia feminicida (ver Tabla 3). Para todas las formas de violencia feminicida percibida, los médicos supervisores del servicio social fueron citados con un $71.8 \%$ como la fuente del abuso.
Tabla 3.

Tipo de Violencia Feminicida identificados en la Centros de Salud del Estado de Guanajuato.

\begin{tabular}{lc}
\hline $\begin{array}{l}\text { Situaciones de Riesgo } \\
\text { en el Centro de Salud }\end{array}$ & Mujeres MPSS* $(\mathbf{n}=\mathbf{6 0})$ \\
\hline Inseguridad (\%) & 30 \\
Vida en Peligro (\%) & 50 \\
Intento de Irrupción (\%) & 20 \\
Tentativa de Acoso Sexual (\%) & 30
\end{tabular}

Fuente: Entrevista dirigida a médicas pasantes. Los datos son expresados como media \pm desviación estándar. a: El total es mayor porque algunas pasantes reportaron más de una forma de violencia. *MPSS: Médicos Pasantes de Servicio Social.

Tabla 4.

Regresión Lineal de los Tipos de Violencia Feminicida y Situaciones de Riesgo en la Centros de Salud del Estado de Guanajuato.

\begin{tabular}{|c|c|c|c|}
\hline $\begin{array}{l}\text { Variable dependiente: Genero R2 ajustada: .966 } \\
\mathrm{p}<.001\end{array}$ & B & $\mathrm{t}$ & $\mathrm{p}$ \\
\hline Inseguridad en el CS & -.476 & -6.78 & .78 \\
\hline Vida en Peligro & -.943 & -13.86 & .96 \\
\hline Intento de Irrupción & 1.55 & .81 & $.00^{*}$ \\
\hline Tentativa de Acoso Sexual & 2.55 & 3.55 & $.00^{*}$ \\
\hline Violencia Psicológica & 2.61 & 3.10 & $.00^{*}$ \\
\hline Violencia Económica & .21 & .42 & .69 \\
\hline Violencia Física & 1.03 & .29 & .61 \\
\hline Violencia Sexual & .35 & .27 & .77 \\
\hline Violencia Laboral & .87 & .40 & .32 \\
\hline Violencia Docente & 1.48 & 1.00 & .41 \\
\hline
\end{tabular}

Nota: $\mathrm{B}=$ coeficientes beta no estandarizados ${ }^{*} \mathrm{p}<.05$

Como se muestra en la Tabla 4, en cuanto a los efectos condicionales, la regresión lineal explica cómo influye el género de manera positiva y significativa en la presencia de intento de irrupción al CS, la tentativa de acoso y la violencia psicológica.

\section{Violencia Feminicida:} Resultados Cualitativos

Las MPSS describieron 16 casos de acoso o violencia. Se percibió que estos eventos representaban un comportamiento normalizado y se basaban en suposiciones. Comúnmente incluían 
invasiones del espacio personal, insinuaciones sexuales y miedo a las consecuencias refiriendo lo siguiente:

"Toda la cirugía el doctor estaba tocando mi cintura, acariciándome, estaba a punto de golpearlo, me molestó"

"Un doctor se me insinuó de forma sexual, sentí que era normal y que no podía decirle a nadie"

"No se lo dije a nadie porque no quiero tener una mala reputación, pero me di cuenta de que todos saben que acosa y es normal"

"Pensé que me metería en más problemas si lo contaba, el doctor podría afectar mi calificación final del servicio social"

"Y me dijo que criar una familia es algo exclusivo de las doctoras, no entendí que quiso decir"

"Hacía bromas sexuales de manera muy frecuente, que me incomodaron mucho, una vez lloré."

"Tuve que abandonar mi centro de salud porque ya no soportaba más."

El 100\% de las estudiantes que percibieron haber sufrido alguna forma de violencia feminicida en su centro de salud no informaron ni denunciaron con alguna autoridad. Las encuestadas, en el 90\% de los casos, no denunciaron ya que pensaron que no lograrían nada al denunciar la violencia percibida. El 10\% de las MPSS afirmaron que no informaron sobre el acoso porque no querían pensar más sobre lo que ocurrió.

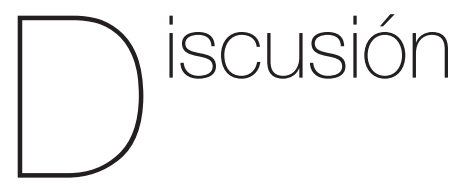

Se encontró una alta prevalencia de violencia y situaciones de riesgo en una muestra de MPSS. Las características sociodemográficas de las mujeres estudiadas concuerdan con otros estudios donde se refiera que la segunda década de la vida es un factor de riesgo para ser violentada (Vives-Cases et al., 2009). La escolaridad es un elemento clave que Lee (2008) sugiere podría evitar ser víctima de la violencia feminicida, sin embargo, en este estudio las mujeres fueron violentadas por sus autoridades docentes o por personas que son originarias de la comunidad donde fueron asignadas, acorde a lo que fundamentan Martínez, O'Brien y Hebl (2017) además de Gill y Febbraro (2013), pero estos últimos en el contexto militar. La discriminación y la violencia que reflejan los resultados de esta investigación es una condición particular ya reportada por otros autores en estudiantes de Medicina, mencionando además que estas conductas son percibidas por los estudiantes como normales (Cubillos \& Sepúlveda, 2013). En nuestros resultados las MPSS tenían 2.5 veces más probabilidad de sufrir intento de irrupción en el CS, violencia sexual y psicológica. Algunas alumnas describieron toques incómodos y la invasión del espacio personal por parte de los médicos a cargo. Las MPSS refirieron varias barreras para informar, la percepción del acoso como "normal", el miedo a la victimización, las repercusiones académicas y que no lograrían nada al denunciar. 
Cobo (2011) afirma que la violencia masculina exhibe una de las caras de la configuración de la desigualdad entre unas y otros, resultado de las relaciones de poder y de autoridad que han inclinado la balanza patriarcal hacia los hombres, lo que explica que estos tengan la potestad de violentar a las mujeres, a cualquier mujer, desde la posición de poderío y privilegio que ostentan en términos de género, la cual se potencia cuando se suman otros atributos de jerarquía como la clase social, la raza, la pertenencia étnica o la adscripción a grupos. Esto se evidenció en el grupo de mujeres estudiadas.

No debemos ignorar que las agresiones que sufren las mujeres tienen graves efectos en su vida, tanto físicas como emocionales. Según la Encuesta Nacional sobre la Dinámica de las Relaciones en los Hogares (ENDIREH, 2016), realizada en México, el 8\% de las mujeres que han sufrido violencia ha pensado en suicidarse y el 3.4\% lo ha intentado. Estas cifras ponen de manifiesto que el problema de la violencia contra las mujeres no ha disminuido; así, según esta encuesta, de los 46.5 millones de mujeres mexicanas de 15 años o más que residen en el país, 30.7 millones (66.1\% en promedio) han sufrido al menos un incidente de violencia: $49 \%$ violencia emocional, seguida por un $41.3 \%$ que declaró haber sufrido violencia sexual; 34\% física y 29\% económica, patrimonial o discriminación laboral a lo largo de su vida, en al menos un ámbito, y ejercida por cualquier agresor. Lo anterior quiere decir que en México casi 7 de cada 10 mujeres han sufrido violencia, la mayoría del tipo emocional y sexual.

Como género, las mujeres en México estamos sometidas en grados diversos a poderes de exclusión, rechazo, discriminación y explotación de género, de tipo estructural, por ser mujeres. Dichas formas de opresión están presentes con peculiaridades en todo el país, en todas las edades, las clases sociales, los grupos étnicos y en todos los órdenes y esferas de la vida privada y pública.

Las mujeres que se evaluaron en este estudio se ubican en el último año de la carrera de Medicina y sus agresores fueron en su mayoría docentes, lo cual concuerda parcialmente con otros estudios donde se refiere abuso sobre las estudiantes de Medicina en un porcentaje alto, pero en semestres iniciales de la carrera y los principales agresores son sus pares (Maida, Herskovic, Pereira, Salinas-Fernández, \& Esquivel, 2006). Un estudio de consenso de las Academias Nacionales de Ciencia, Ingeniería y Medicina de 2018 describió la frecuencia alarmante de acoso de género, atención sexual no deseada y coerción sexual en la ciencia y la Medicina académica, lo cual no concuerda con nuestros resultados, ya que la violencia más común reportada en esta muestra de mujeres fue la violencia física y económica, mientras que la sexual se reportó como la menos frecuente; sin embargo la tentativa de acoso tuvo una frecuencia del 30\%.

Los hallazgos referentes a la violencia física reportada en este estudio reafirman el abuso de autoridad como lo describe Sreeramareddy, Shankar, \& Binu (2007), donde describen que la violencia laboral incluye carga de trabajo de más de 12 horas, además de trabajo administrativo solicitado por sus superiores generando reportes, formatos, censos y bitácoras de manera mensual. 
El incentivo monetario hacia las pasantes fue condicionado al desempeño en el trabajo, ejerciendo violencia económica, lo cual resulta contradictorio ya que los reglamentos sobre servicio social, en el apartado de derechos y obligaciones fundamentados en la Ley Orgánica de la Administración Pública, la Ley General de Salud, la Ley Reglamentaria del Artículo 5 Constitucional, el Reglamento Interior de la Secretaria de Salud y el Plan Nacional de Desarrollo 2013-2018, señalan que los médicos pasantes recibirán $\$ 3,000.00$ y $\$ 2,000.00$ si se encuentran asignados a un centro de salud rural o urbano respectivamente.

El tipo de violencia feminicida más frecuentemente percibido en este estudio fue la violencia física, lo que concuerda con lo descrito por Boyle y McKenna (2017) donde detallan que un 32.6\% de estudiantes estuvieron expuestos al menos a un acto de violencia en el lugar de trabajo, y el 56\% de ellos eran mujeres. El abuso académico fue reportado por el $43 \%$ de las pasantes, consistente con estudios previos donde reportan intimidación no verbal, como evitar el contacto visual con los estudiantes, anular su trabajo (Siller, Tauber, Komlenac, \& Hochleitner, 2017; Thomas \& Burk, 2009) e ignorarlos (Curtis \& Bowen, 2007). Los estudiantes también han recibido cantidades irrazonables de tareas (Lash, Kulakaç, Buldukoglu, \& Kukulu, 2006), asignándoles demasiada responsabilidad teniendo en cuenta sus habilidades (Curtis \& Bowen, 2007; Thomas \& Burk, 2009), y se les han establecido tareas demandantes sin suficiente orientación (Curtis \& Bowen, 2007; Sandelin, 2007). Deben tenerse en cuenta algunas limitaciones al interpretar los resultados de este estudio. En primer lugar, estos resultados no pueden generalizarse debido al pequeño tamaño de la muestra. Sin embargo, hay coherencias entre nuestros resultados e investigaciones previas.

Las Escuelas y Facultades de Medicina en conjunto con las autoridades deberán realizar acciones de impacto en estos grupos de mujeres, para tomar decisiones importantes respecto a la violencia a la que han sido expuestas generación tras generación. Las autoridades competentes deben proporcionar el marco referencial necesario para reducir y eliminar de manera completa los diferentes tipos de violencia que, tanto docentes, supervisores y población de las comunidades rurales y urbanas, ejercen sobre las MPSS de la carrera de Medicina. Los Institutos de las Mujeres y otros mecanismos afines aún no intervienen con suficiencia en el registro de la violencia, aunque, en su mayoría, despliegan algún programa parcial de atención a la violencia y, desde el Instituto Nacional de las Mujeres hasta los de las entidades federativas, realizan campañas para visibilizar o denunciar la violencia; sin embargo, no es suficiente.

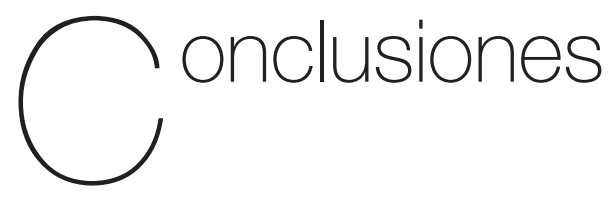

Las médicas pasantes de servicio social son violentadas por diferentes acciones y/o omisiones, lo que causa sufrimiento psicológico, físico, laboral y económico. El índice de violencia contra las mujeres va en aumento. En las mujeres MPSS son evidentes las agresiones violentas, sugiriendo que los CS no tienen 
medidas de seguridad apropiadas para resguardar a estas mujeres. La violencia feminicida en las escuelas de Medicina constituye un problema de derechos humanos, salud pública y académico debido a sus efectos en la salud física y mental de las estudiantes y sus consecuencias en su trayectoria académica. Es indispensable generar políticas públicas que desnaturalicen y hagan visible la violencia feminicida, así como modificar las relaciones de género asimétricas en las escuelas de Medicina de las diversas universidades, en los CS y los hospitales. Es muy importante modificar la importancia del cuerpo y la sexualidad de las mujeres y otorgarles el estatus de sujetos con derechos es ineludible. Crear centros de quejas y unidades de ayuda especializadas y confiables que apoyen a las estudiantes con soluciones y estrategias efectivas, garantizando la confidencialidad sin temor a represalias, además de hacer visible la magnitud e importancia del problema. Habrá que insistir en crear procedimientos que incluyan que las mujeres víctimas de la violencia deberán contar con un refugio seguro que garantizará: hospedaje, alimentación, vestido, servicio médico, asesoría jurídica, apoyo psicológico y diversos programas de reinserción a la sociedad. La atención integral, desde la perspectiva de género, deberá estar a cargo de profesionales, y las mujeres deberán recibir capacitación laboral y tener acceso a bolsa de trabajo. Asimismo, se realizarán programas reeducativos integrales a fin de que las mujeres logren estar en condiciones de participar plenamente en la vida pública, social y privada. El conjunto de acciones se inscribe en la vía de lograr el empoderamiento de las mujeres y la construcción de su condición ciudadana, con el pleno ejercicio de sus derechos.
A lo largo de la vida académica, durante muchos años, las médicas son objeto de agresiones sexuales, físicas, emocionales, verbales, patrimoniales y laborales. Los grupos académicos consideran natural esta violencia, culpabilizando y señalando a las mujeres como víctimas propiciatorias de los delitos en su contra, al mismo tiempo que exoneran a los hombres; en ocasiones se frivoliza y es parte del humor cultivado socialmente.

Las investigadoras esperamos que este estudio permita que el personal académico, administrativo y de atención médica reflexione y tome conciencia de la violencia feminicida que experimentan las MPSS, y que se tomen medidas diseñando intervenciones focalizadas para la prevención de la violencia contra las mujeres, así como diseñar y promover la aplicación de intervenciones preventivas enfocadas a las mujeres como sujetas de derechos, para la desnaturalización de la violencia.

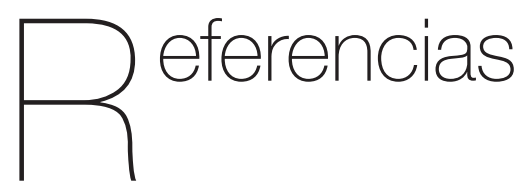

Alzahrani, H.A. (2012). Bullying among medical students in a Saudi medical school. BMC Research Notes, 1(5), 335-340.

Artículo No. 5. Constitución Política de los Estados Unidos Mexicanos. (2013). Últimas reformas. Recuperado de http://www. diputados.gob.mx/LeyesBiblio/pdf/1.pdf

Ato, M., López, J.J., \& Benavente, A. (2013). Un sistema de clasificación de los diseños 
de investigación en psicología. Anales de Psicología, 29(3), 1038-1059.

Benya, F.F., Widnall, S.E., \& Johnson. PA. (2018). Sexual Harassment of Women: Climate, Culture. Consequences in Academic Sciences, Engineering, Medicine. Washington, DC, USA: National Academies Press.

Bonilla-Neira, L., Gómez-Meneses, C., \& Godoy-Pinilla, J.C. (2019). Investigaciones y estrategias comunicativas frente a la violencia contra las mujeres en ámbitos universitarios. Una revisión. Informes Psicológicos, 19(1), 141-162.

Boyle, M. \& McKenna, L. (2017). Paramedic student exposure to workplace violence during clinical placements. A crosssectional study. Nurse Education in Practice, 1(22), 93-97.

Bullen, M. \& Diez, C. (coords.) (2008). Retos teóricos y nuevas prácticas. Serie, XI Congreso de Antropología de la FAAEE, Donostia, Ankulegi Antropologia Elkartea. Recuperado de https://core.ac.uk/ download/pdf/11500603.pdf

Castro, R. (2014). Génesis y práctica del habitus médico autoritario en México. Revista Mexicana Sociología, 76(2), 167-197.

Cubillos, C. \& Sepúlveda, L. (2013). Discriminación y violencia contra los estudiantes de medicina de la Universidad de Caldas. Investigación en Educación Médica, 2(1), 37-41.

Curtis, J.I. \& Bowen, A.R. (2007) You have no credibility: nursing students' experiences of horizontal violence. Nurse Education in Practice, 7(3), 156-163.
Cobo, R. (2011). Hacia una nueva política sexual. Las mujeres ante la reacción patriarcal. Madrid: Los Libros de la Catarata.

Diario Oficial de la Federación. (2011). Lineamientos del Programa de Servicio Social. Recuperado de http://www. diputados.gob.mx/LeyesBiblio/pdf/1.pdf

Encuesta Nacional sobre la Dinámica de las Relaciones en los Hogares (ENDIREH) (2016). Principales resultados, INEGl. Recuperado de http://www.beta. inegi.org.mx/contenidos/proyectos/ enchogares/especiales/endireh/2016/doc/ endireh2016_presentacion_ejecutiva.pdf

Fernández-Moreno, S. (2007). La violencia de género en las prácticas institucionales de salud: afectaciones del derecho a la salud y a las condiciones de trabajo en salud. Revista Gerencia Políticas de Salud, 6(12), 52-76.

Fnais, N., Soobiah, C., Chen, M., et al. (2014). Harassment and discrimination in medical training. Academic Medicine, 89(5), 817-27.

Frank, E., Carrera, J.S., Stratton, T., Bickel, J., \& Nora, L. M. (2006). Experiences of belittlement and harassment and their correlates among medical students in the United States: longitudinal survey. BMJ, 333(7570), 682-685.

Gill, R., \& Febbraro, A.R. (2013). Experiences and Perceptions of Sexual Harassment in the Canadian Forces Combat Arms. Violence Against Women, 19(2), 269-287.

Hasty, B.N., Miller, S.E., Bereknyei-Merrell, S., Lin, D.T., Shipper, E.S., \& Lau, J.N. (2018). Medical student perceptions of a mistreatment program during the surgery 
clerkship. American Jornual of Surgery, 215(4), 761-766.

Izquierdo, J. (1998). Los órdenes de la violencia: especie, sexo y género. El sexo de la violencia: género y cultura de la violencia, 1(1), 1-21.

Lagarde, M. (2001). Género y feminismo. Desarrollo humano y democracia. Madrid: Horas y Horas.

Lagarde, M. (2006). Presentación a Violencia Feminicida en 10 entidades de la República Mexicana, Violencia Feminicida en Baja California, Chiapas, Distrito Federal, Chihuahua, Estado de México, Guerrero, Morelos, Oaxaca, Veracruz, Sonora. Geografía de la Violencia Feminicida en la República Mexicana.

Lash, A.A., Kulakaç, Ö., Buldukoglu, K., \& Kukulu K. (2006) Verbal abuse of nursing and midwifery students in clinical settings in Turkey. Journal of Nursing Education, 45(10), 396-403.

Lee, J. (2008). Survivors of Gendered Violence in the Feminist Classroom. Violence Against Women, 14(12), 1451-1464.

López, J., González, M. \& Velasco, M. (2004). Servicio social de medicina en México. Factibilidad del cumplimiento académico en el área rural. Revista de la Facultad de Medicina de la UNAM, 47(5), 123-125.

Maida, M., Herskovic, V., Pereira, A., SalinasFernández, L., \& Esquivel, C. (2006). Percepción de conductas abusivas en estudiantes de medicina. Revista Médica de Chile, 134(12), 1516-1523.

Martínez, L.R., O'Brien, K.R., \& Hebl, M.R. (2017). Fleeing the Ivory Tower: Gender
Differences in the Turnover Experiences of Women Faculty. Journal of Womens Health (Larchmt), 26(5), 580-586.

Mavis, B., Sousa, A., Lipscomb, W., \& Rappley, M. (2014). Learning about medical student mistreatment from responses to the medical school graduation questionnaire. Academic Medicine, 89(5), 705-711.

Moreno-Tetlacuilo, L. M., Quezada-Yamamoto, H., Guevara-Ruiseñor, E. S., Ibarra-Araujo, N., Martínez-Gatica, N. L., \& PedrazaMoreno, R. (2016). Gender-based relations and mistreatment in medical schools: A pending agenda in Mexico and the world. Gaceta Médica de México, 152(6), 812-818.

Nagasawa, T., Nomura, K., Takenoshita, S., Hiraike, H., Tsuchiya, A., Ohkubo, T., \& Okinaga, H. (2019). Scale Development on Perception of Academic Harassment among Medical University Faculties. Nihon Eiseigaku Zasshi, 74(0), 18-33.

Nora, L., McLaughlin, M., Fosson, S., et al. (2007) Gender discrimination and sexual harassment in medical education. Academic Medicine, 77(12), 1226-1234.

Ramírez, O., Hamui, A., Reynaga, J., Varela, M., Ponce de León, M., Díaz, A., \& Valverde, I. (2012). Condiciones de trabajo de los médicos pasantes mexicanos durante el servicio social. Perfiles Educativos, 34(138), 1-12.

Ramírez, M. (2009). Expectativas profesionales de internos de medicina y su inclinación por la atención primaria de salud. Anales de la Facultad de Medicina, 69(3), 176-181.

Rees, C., \& Monrouxe, L. (2011). A morning since eight of just pure grill»: a multischool 
qualitative study of student abuse. Academic Medicine, 86(11), 1374-82.

Ritu, G. \& Febbraro, A. (2013). Experiences and Perceptions of Sexual Harassment in the Canadian Forces Combat Arms. Violence Against Women, 19(2), 269-287.

Sandelin, P. (2007). Kertomuksia psyykkisestä väkivallasta terveydenhuollon työ- ja opiskeluyhteisöissä (Narrations of mental violence in workplaces and study communities in the health care sector). (Doctoral dissertation). Acta Universitatis Ouluensis D Medica, 931, 1-66.

Secretaría de Salud (1982). Bases para la instrumentación del Servicio Social de las Profesiones para la Salud. Recuperado de http://www.diputados.gob.mx/ LeyesBiblio/pdf/1.pdf

Siller, H., Tauber, G., Komlenac, N. \& Hochleitner, M. (2017). Diferencias y similitudes de género en las experiencias de maltrato de estudiantes de Medicina por parte de varios grupos de perpetradores. BMC Medical Education, 17(1), 134-139
Silver, H. (1982). Medical students and medical school. JAMA, 247(3), 309-310.

Sreeramareddy, C., Shankar, P., \& Binu, V. (2007). Psychological morbidity, sources of stress and coping strategies among undergraduate medical students of Nepal. BMC Medical Education, 7(26), 1-8.

Thomas, S.P., \& Burk R. (2009). Junior nursing students' experiences of vertical violence during clinical rotations. Nursing Outlook, 57(4), 226-231.

Vanlneveld, C.H., Cook, D.J., Kane, S.L., \& King, D. (1996). Discrimination and abuse in internal medicine residency. The Internal Medicine Program Directors of Canada. Journal of General Internal Medicine, 11(1), 401-405.

Vives-Cases, C., Álvarez-Dardet, C., GilGonzález, D., Torrubiano-Domínguez, J., Rohlfs I., \& Escribà-Agüir, V. (2009). Perfil sociodemográfico de las mujeres afectadas por violencia del compañero íntimo en España. Gaceta Sanitaria, 23(5), 410-414. 